\title{
Research on the Parameter Equation of Space Curve's Projection on the Flat Surface Xihe Liu ${ }^{1, a}$, Yijia Gao ${ }^{1, b}$
}

${ }^{1}$ Graduate School of Environment and Information Sciences, Yokohama National University, Yokohama, 240-8501, Japan

${ }^{a}$ email, ${ }^{b}$ email

Keywords: Parameter equation, Space curve, Projection on the flat surface

\begin{abstract}
It is an important content to study the parameter equation of the projection of space curve in plane. In this paper, by means of lemma and theorem, the method of finding the projective parameter equation of space curve on the plane is given and verified by examples to provide some references for the relative researchers.
\end{abstract}

\section{Introduction}

Curve is one of the main objects of differential geometry. Intuitively, the curve can be regarded as the trajectory of the motion of the particles in space. Differential geometry is the study of geometry using calculus. In order to be able to apply the knowledge of calculus, we cannot consider all curves, or even consider continuous curves, because continuity is not necessarily differentiable. This requires us to consider differentiable curves. But the differentiable curve is not good, because there may be some curves, at some point in the tangent direction is not determined, so that we could not start from the tangent line, which we need to study this kind of derivative everywhere is not zero, we call them regular curve. The regular curve is the main object of classical curve theory. Any continuous line is called a curve. It includes straight line, broken line, line segment, arc and so on. The curve that turns everywhere has generally the length of infinity and the area of zero, and then the curve itself is a space. One of the main objects of differential geometry. Intuitively, the curve can be regarded as the trajectory of the motion of the particles in space. A more rigorous definition of a curve is the mapping. Sometimes the mapped image is called a curve. Specifically, let Oxyz be the Cartesian coordinate system in Euclidean space and is the path of the point on the curve $\mathrm{C}$, so there is. The upper form is called the parametric equation of the curve $\mathrm{C}$, and $\mathrm{t}$ is called the parameter of the curve $\mathrm{C}$, and the positive direction of the curve $\mathrm{C}$ is determined naturally according to the increasing direction of the parameter. The regular curves are often discussed in the theory of curves. The functions of their coordinate functions $\mathrm{x}(\mathrm{t}), \mathrm{y}(\mathrm{t}), \mathrm{z}(\mathrm{t})$ derivatives are continuous, and curves of any $\mathrm{t}$ are not zero at the same time. For regular curves, the arc length $\mathrm{s}$ is always taken as a parameter, which is called the natural parameter or the arc length parameter. In the analysis of analytic geometry and mathematics, often require the curve projection equation of curve, in many books, the projection curve equation of space curve in the plane is mostly expressed by general equations. In order to facilitate the application, researchers should find the parameter equation of projection curve.

\section{Lemma and Theorem}

Lemma. Assume the parameter equation of space curve $\Gamma$ is:

$\left\{\begin{array}{l}x=\varphi(t) \\ y=\psi(t) . \\ z=\omega(t)\end{array}\right.$

Then, the cylindrical parameter equation which take $\Gamma$ as the alignment and whose generatrix is parallel to $s=\{l, m, n\}$ is: 


$$
\left\{\begin{array}{c}
x=\varphi(t)+l k \\
y=\psi(t)+m k \\
z=\omega(t)+n k
\end{array}\right.
$$

In the above equation, $\mathrm{t}$ and $\mathrm{k}$ are parameters.

Proof: Assume the point $\left(x_{0}, y_{0}, z_{0}\right)$ corresponds to $t=t_{0} .\left(\varphi\left(t_{0}\right), \psi\left(t_{0}\right), \omega\left(t_{0}\right)\right)$ is one point of the curve $\Gamma$. Then, the curve parameter parallel to $s=\{l, m, n\}$ is:

$$
\left\{\begin{array}{c}
x=\varphi\left(t_{0}\right)+l k \\
y=\psi\left(t_{0}\right)+m k \text { (k is parameter). } \\
z=\omega\left(t_{0}\right)+n k
\end{array}\right.
$$

If we transform $t_{0}$ to $t$. With the change of $t$, the equation

$$
\left\{\begin{array}{c}
x=\varphi(t)+l k \\
y=\psi(t)+m k \\
z=\omega(t)+n k
\end{array}\right.
$$

represents the family of parallel lines which intersects with $\Gamma$. Therefore,

$$
\left\{\begin{array}{c}
x=\varphi(t)+l k \\
y=\psi(t)+m k \\
z=\omega(t)+n k
\end{array}\right.
$$

is the cylindrical parameter equation which take $\Gamma$ as the alignment and whose generatrix is parallel to $s=\{l, m, n\}$.

Theorem. Assume the parameter equation of space curve $\Gamma$ is:

$$
\left\{\begin{array}{l}
x=\varphi(t), \\
y=\psi(t), \\
z=\omega(t)
\end{array}\right.
$$

then, the parameter equation of space curve's projection on the flat surface $\pi, A x+B y+C z+D=0$ is:

$$
\left\{\begin{array}{l}
\mathrm{x}=\varphi(t)-A \Phi(t) \\
y=\psi(t)-B \Phi(t) \\
z=\omega(t)-C \Phi(t)
\end{array}\right.
$$

In the above formulation, $\Phi(t)=\frac{A \varphi(t)+B \psi(t)+\mathrm{C} \omega(t)+\mathrm{D}}{A^{2}+B^{2}+C^{2}}$.

Proof: From the Lemma, the cylindrical parameter equation which take $\left\{\begin{array}{l}\mathrm{x}=\varphi(t), \\ y=\psi(t), \\ z=\omega(t),\end{array}\right.$ as the alignment and whose generatrix is parallel to $S=\{A, B, C\}$ is:

$$
\left\{\begin{array}{l}
\mathrm{x}=\varphi(t)+A k \\
y=\psi(t)+B k \\
z=\omega(t)+C k
\end{array}\right.
$$

In the above formulation, $\mathrm{t}$ ank $\mathrm{k}$ are parameters. Thus, the alignment of the cylinder is parallel to the normal vector $\{A, B, C\}$ of $\pi, A x+B y+C z+D=0$.

Then, $A[\varphi(t)+A k]+B[\psi(t)+B k]+C[\omega(t)+C k]+\mathrm{D}=0$. 
and $k=-\frac{A \varphi(t)+B \psi(t)+\mathrm{C} \omega(t)+\mathrm{D}}{A^{2}+B^{2}+C^{2}}$.

Let $\Phi(t)=\frac{A \varphi(t)+B \psi(t)+C \omega(t)+\mathrm{D}}{A^{2}+B^{2}+C^{2}}$,

Then, $k=-\Phi(t)$. We plug it into the equation,

$\left\{\begin{array}{l}\mathrm{x}=\varphi(t)+A k, \\ y=\psi(t)+B k, \\ z=\omega(t)+C k,\end{array}\right.$

Therfore, the projection parameter equation of space curve $\Gamma$ on the flat surface $\pi$ is:

$\left\{\begin{array}{l}\mathrm{x}=\varphi(t)-A \Phi(t) \\ y=\psi(t)-B \Phi(t), \\ z=\omega(t)-C \Phi(t)\end{array}\right.$

\section{Application Examples}

Example 1. Seek the line projection parameter equation of the line

$\left\{\begin{array}{l}x=9 t, \\ y=7 t-1, \\ z=10 t-4,\end{array}\right.$

on the plane of $4 \mathrm{x}-\mathrm{y}+\mathrm{z}-1=0$.

Solution: According to the line general equation, we know

$A=4, B=-1, C=1, D=-1$,

and the $\varphi(t)=9 t, \psi(t)=7 t-1, \omega(t)=10 t-4$,

so, we have:

$$
\begin{aligned}
& \Phi(t)=\frac{4 \times 9 t-1 \times(7 t-1)+1 \times(10 t-4)-1}{4^{2}+(-1)^{2}+1^{2}} \\
& =\frac{13}{6} t-\frac{2}{9} .
\end{aligned}
$$

According to the above theorem, the line projection parameter equation is;

$$
\left\{\begin{array}{l}
x=9 t-4 \times\left(\frac{13}{6} t-\frac{2}{9}\right), \\
y=7 t-1+\left(\frac{13}{6} t-\frac{2}{9}\right), \\
z=10 t-4-\left(\frac{13}{6} t-\frac{2}{9}\right),
\end{array}\right.
$$

namely,

$$
\left\{\begin{array}{l}
x=\frac{1}{3} t+\frac{9}{8}, \\
y=9 \frac{1}{6} t-\frac{11}{9}, \\
z=7 \frac{5}{6} t-3 \frac{7}{9},
\end{array}\right.
$$


Example 2. Seek the projection parameter equation of the space curve

$C:\left\{\begin{array}{l}x=3 \cos \theta, \\ y=4 \sin \theta,(0 \leq \theta \leq 2 \pi) \\ z=5,\end{array}\right.$

on the plane of $x+y+z+1=0$.

Solution: To construct the perpendicular of the plane passing through the point $(x(\theta), y(\theta), z(\theta))$ of the curve $C$. The perpendicular equation is:

$\frac{x-3 \cos \theta}{1}=\frac{y-4 \sin \theta}{1}=\frac{z-5}{1}$.

Then, it is transformed into parameter model:

$\left\{\begin{array}{c}x=3 \cos \theta+s, \\ y=4 \sin \theta+s, . \\ z=5+s,\end{array}\right.$

We plug it into the known plane equation to seek $S$, recorded as $S_{0}$.

$s_{0}=\frac{3 \cos \theta+4 \sin \theta+5+1}{3}$.

Then, we can obtain the coordinate of the projection point.

$\left\{\begin{array}{c}x=3 \cos \theta+s_{0}, \\ y=4 \sin \theta+s_{0}, \\ z=5+s_{0},\end{array}\right.$

It is the solution parameter equation of the space curve projection.

\section{Conclusions}

Most of the analytic geometry textbooks give the projection equation of the space curve on the coordinate plane. However, the projection equation of the space curve on the general plane has never been mentioned in the textbooks. This paper provides the method of finding the projective parameter equation of space curve on the general plane to make up for the shortcomings of the teaching materials.

\section{References}

[1] Liu Xingyuan, He Yiju, The Teaching Discussion About Parameter Equation of Space Curve [J]. Journal of Shaoyang University (Natural Science Edition), 2011, 8(1): 26-28.

[2] Wang Shuying, Design of Sine and Cosine Curve Drawing Model by Projection Principle [J]. Journal of Nanjing Institute of Industry Technology, 2014, 14(4): 13-16+28.

[3] Ding Diankun, Bian Pingyong, The Parameter Equation of Space Curve's Projection on the Flat Surface [J]. College Mathematics, 2006, 22(3): 147-150.

[4] Zhang Xiaoyan, Zhu Meng, Dong Benzhi, Offset algorithm of a third-order NURBS curve based on projection [J]. Wood Processing Machinery, 2016, 27(2): 29-32. 